\title{
Nonlinear effects of particle shape angularity in sheared granular media
}

\author{
Emilien Azéma, ${ }^{1, *}$ Nicolas Estrada, ${ }^{2, \dagger}$ and Farhang Radjai ${ }^{1, \ddagger}$ \\ ${ }^{1}$ LMGC, Université Montpellier 2-CNRS, Place Eugène Bataillon, 34095 Montpellier cedex 05, France \\ ${ }^{2}$ Departamento de Ingeniería Civil y Ambiental, Universidad de Los Andes, Bogotá, Colombia
}

(Dated: September 19, 2012)

\begin{abstract}
We analyze the effects of particle shape angularity on the macroscopic shear behavior and texture of granular packings simulated by means of the contact dynamics method. The particles are regular polygons with an increasing number of sides ranging from 3 (triangles) to 60. The packings are analyzed in the steady shear state in terms of their shear strength, packing fraction, connectivity, and fabric and force anisotropies, as functions of the angularity. An interesting finding is that the shear strength increases with angularity up to a maximum value and saturates as the particles become more angular (below six sides). In contrast, the packing fraction declines towards a constant value, so that the packings of more angular particles are looser but have higher shear strength. We show that the increase of the shear strength at low angularity is due to an increase of both contact and force anisotropies, and the saturation of the shear strength for higher angularities is a consequence of a rapid fall-off of the contact and normal force anisotropies compensated by an increase of the tangential force anisotropy. This transition reflects clearly the rather special geometrical properties of these highly angular shapes, implying that the stability of the packing relies strongly on the side-side contacts and the mobilization of friction forces.
\end{abstract}

\section{INTRODUCTION}

Granular materials composed of particles of complex shape are common in nature and also in various fields of science and engineering. Some examples are angularshaped particles of soils and rocks, elongated or platy particles of pharmaceutical products and non-convex particles of metallurgical and sintered powders. These shape characteristics strongly affect the rheology and texture of granular materials. This has been recently evidenced by a number of numerical and experimental studies carried out using angular particles [1-12] and by a number of investigations that have focused on other important characteristics such as elongation [13-20] or non-convexity $[21,22]$. The existing research results suggest that the effect of shape parameters is often nonlinear and counterintuitive as in the case of the unmonotonic relation between the elongation of the particles and the packing fraction $[13,14,18]$.

Hence, in order to obtain a clear picture of the complex behavior exhibited by real granular materials, it is crucial to understand and quantify the effects of particle shape. However, this is not an easy task, which is why systematic studies on the subject are scarce. One of the underlying issues is that it is difficult to control particle shape in experiments. Moreover, introducing particle shape in numerical simulations with discrete element methods gives rise to various technical difficulties, both geometrical and computational. One example of these difficulties involves contact detection and force calculation between particles of arbitrary shape [7, 10, 23-26].

\footnotetext{
*Electronic address: emilien.azema@univ-montp2.fr

${ }^{\dagger}$ Electronic address: n.estrada22@uniandes.edu.co

¥Electronic address: farhang.radjai@univ-montp2.fr
}

The aim of this work is to explore the influence of the degree of angularity of the particles on the mechanical behavior of sheared granular packings. We employ the contact dynamics method to simulate large two-dimensional packings of polydisperse regular polygonal particles. We construct different packings, each of them made up of particles with a given number of sides in the range varying from 3 (triangles) to 60 . We also simulate a packing of disks, which may be considered as polygons of an infinite number of sides. Hence, the angularity, defined as the exterior angle of polygons, varies from 0 for disks to $2 \pi / 3$ for triangles.

The packings are analyzed in the steady state in terms of their shear strength, packing fraction, connectivity, and fabric and force anisotropies, as functions of the angularity. A pending issue that we would like to address in this paper is whether the packing of disks has a singular behavior compared to the packings of polygons. This is the case if a discontinuous change (within our statistical precision) is observed for a packing property, e.g. the shear strength or packing fraction, between the packing of disks and the packing of polygons of 60 sides, which are least angular polygons in our simulations. In a similar vein, it is not obvious whether packings composed of particles of the lowest numbers of sides, i.e. triangles and squares, are special as compared to those of larger numbers of sides whose behavior is expected to be described by the angularity parameter as deviation from circular shape.

In the following, we introduce in Section II the numerical approach, system characteristics, and loading parameters. In Section III, we focus on the evolution of shear strength and packing fraction with angularity. The microstructure is analyzed in Section IV in terms of connectivity, and contact and force anisotropies. The final section presents the concluding remarks and a summary of the most salient results. 


\section{MODEL DESCRIPTION}

\section{A. Numerical method}

The simulations were carried out by means of the contact dynamics (CD) method, which is suitable for large assemblies of undeformable particles. This method emerged from a mathematical formulation of nonsmooth dynamics and the subsequent algorithmic developments by J. J. Moreau and M. Jean [27-38]. The fundamental difference between this method and the common DEM or molecular dynamics (MD) approach lies in the treatment of small length and time scales involved in the dynamics of granular media. In MD-type DEM, pioneered by P. Cundall, the particles are treated as rigid bodies but the contacts between particles are assumed to obey a viscoelastic or plastic behavior in which the local strain variables are defined from the relative particle positions or displacements ([39-52]). The time-stepping schemes used for the numerical integration of the equations of motion imply thus a fine resolution of the small time and length scales involved in contact interactions.

In the CD method, these small scales are neglected and their effects absorbed into contact laws together with a nonsmooth formulation of particle dynamics described at the scale of particle displacements rather than small elastic response times and displacements. The equations of motion are integrated by an implicit time-stepping scheme by taking into account the kinematic constraints resulting from frictional contact interactions. The implicit integration makes the method unconditionally stable. Moreover, since in this method the elastic contact deflections are not resolved, the time step can be larger than that in the molecular dynamics (MD) method where the time step should be small enough to allow for smooth variations of the overlap at the contact points to ensure numerical stability. In CD, an iterative algorithm is used to determine the contact forces and particle velocities simultaneously at all potential contacts. A detailed presentation of the CD method is given in Appendix A for point contact interactions.

The particle shape enters a CD resolution algorithm through the explicit determination of the set of effective contacts at the beginning of a time step. For polygonal particles, two different types of contact can be distinguished: 1) side-vertex and 2) side-side; see Fig. 1. A side-vertex contact is a point contact like that between two disks. In this case, the side coincides with the tangent common line and the local frame is defined with respect to this line. In a detection algorithm, such as the shadow overlap method used in our simulations, a side-vertex contact corresponds to a single corner of a polygon crossing a side of a partner polygon $[23,25,56]$. Obviously, ideal contacts with no interpenetration of the particles $\left(\delta_{n}=0\right)$ would require infinite precision. In all numerical methods, the detection of contact between two bodies consists actually in observing an overlap of the portions of space they occupy, so that $\delta_{n} \leq 0$. These

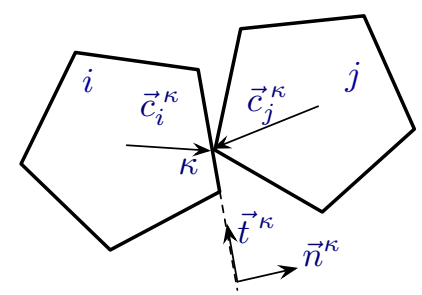

(a)

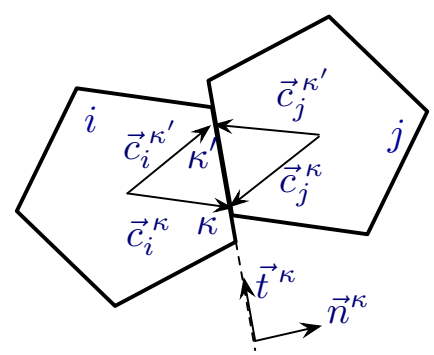

(b)

FIG. 1: Side-vertex contact (a) and side-side contact (b) between two polygonal particles.

overlaps are a simple matter of geometrical precision in the framework of the CD method rather than a strain variable as in MD. The evolution of a granular system by a CD process is as much sensitive to such imperfections as that of a real system to the surface irregularities of real particles.

A side-side contact between two rigid polygons is equivalent to two geometrical constraints and can thus be represented by two distinct point contacts located on the common side, which defines the common tangent line between the two polygons. For this reason, we refer to side-side contacts as double contacts in contrast to sidevertex contacts to which we refer as simple contacts. In practice, a double contact is detected when a double intrusion occurs between two polygons (two vertices of a polygone crossing the same side of another polygon or at least one vertice of each of the two polygons crossing a side of the other polygon). The common tangent line is defined as an intermediate line crossing the overlap zone between the two sides involved in the double contact and the projections of the intruding vertices onto this line are used to define two points representing the double contact. The algorithm is insensitive to the technical details of this choice as long as the intrusions are small compared to particle sizes, i.e. if the neighbor list is frequently updated and the time step is sufficiently small. For example, in our simulations the intrusion never exceeds $1 \%$ of particle diameter.

The two points of a double contact determined by the detection procedure obey Signorini's conditions and Coulomb's friction law; see Appendix A. However, the forces and displacements at the two points are coupled as a result of the rigidity of the particles, which imposes the equality of the sliding velocities. Let $\kappa$ and $\kappa^{\prime}$ be two 
points belonging to a double contact between two polygons, as shown in Fig. 1. The contact frame $(\vec{n}, \vec{t})$ is common to the two point contacts, but the contact vectors $\vec{c}_{i}^{\kappa}$ and $\vec{c}_{j}^{\kappa^{\prime}}$ are different. If both contact points are persistent and nonsliding, the contact normal forces $f_{n}^{\kappa}$ and $f_{n}^{\kappa^{\prime}}$, and tangential forces $f_{t}^{\kappa}$ and $f_{t}^{\kappa^{\prime}}$ may take independent values compatible with Signorini's conditions $\left(f_{n}^{\kappa} \geq 0, f_{n}^{\kappa^{\prime}} \geq 0\right)$ and with Coulomb's law of friction $\left(\left|f_{t}^{\kappa}\right| \leq \mu f_{n}^{\kappa},\left|f_{t}^{\kappa^{\prime}}\right| \leq \mu f_{n}^{\kappa^{\prime}}\right)$. But if one of the two contacts is sliding, then the other contact must be sliding, too, with the equality of the sliding velocities $u_{t}^{\kappa}=u_{t}^{\kappa^{\prime}}$. This condition implies that $f_{t}^{\kappa}$ and $f_{t}^{\kappa^{\prime}}$ are of the same sign so that the sliding status is verified not only at each of the two contact points $\left(f_{t}^{\kappa}= \pm \mu f_{n}^{\kappa}\right.$ and $\left.f_{t}^{\kappa^{\prime}}= \pm \mu f_{n}^{\kappa^{\prime}}\right)$ but also for the double contact, i.e. $f_{t}^{\kappa}+f_{t}^{\kappa^{\prime}}= \pm \mu\left(f_{n}^{\kappa^{\prime}}+f_{n}^{\kappa^{\prime}}\right)$.

Since the equations of dynamics are based on the rigidbody degrees of freedom, the equality of sliding velocities at the two points representing a double contact is in principle correctly calculated if the two points are handled as independent contacts in the iteration process. However, the number of iterations for convergence declines if the equality of the sliding velocities is enforced directly in the iteration process. To do so, Coulomb's friction law for a double contact is implemented as follows:

$$
\begin{cases}u_{t}^{\kappa}>0 & \Rightarrow\left\{\begin{array}{l}
f_{t}^{\kappa}=-\mu f_{n}^{\kappa} \\
f_{t}^{\kappa^{\prime}}=-\mu f_{n}^{\kappa^{\prime}} \text { and } u_{t}^{\kappa^{\prime}}>0 \\
u_{t}^{\kappa}=0 \text { or } u_{t}^{\kappa^{\prime}}=0
\end{array}\right. \\
-\mu f_{n}^{\kappa} \leq f_{t}^{\kappa} \leq \mu f_{n}^{\kappa} \\
u_{t}^{\kappa}<0 & \Rightarrow\left\{\begin{array}{l}
\kappa_{n}^{\kappa^{\prime}} \leq f_{t}^{\kappa^{\prime}} \leq \mu f_{n}^{\kappa^{\prime}} \\
f_{t}^{\kappa}=\mu f_{n}^{\kappa} \\
f_{t}^{\kappa^{\prime}}=\mu f_{n}^{\kappa^{\prime}} \text { and } u_{t}^{\kappa^{\prime}}<0
\end{array}\right.\end{cases}
$$

In practice, the inequalities (1) are implemented in the correction step when solving the local Signorini-Coulomb problem for a double contact between two particles; see Appendix A.

The two points attributed to a double contact and the calculated forces are only intermediate objects. The only physically meaningful forces acting at a double contact are the resultant forces $f_{n}=f_{n}^{\kappa}+f_{n}^{\kappa^{\prime}}$ and $f_{t}=f_{t}^{\kappa}+f_{t}^{\kappa^{\prime}}$. It is easily shown that $f_{n} \geq 0$ and $\left|f_{t}\right| \leq \mu f_{n}$ if the two contact points obey Signorini's conidtions and Coulomb's friction law. Since only the force resultants and relative displacements are material at a double contact, the choice of the two representative points of a double contact is a matter of technical convenience with no real impact on the result.

Ideally, vertex-vertex contacts should never occur, but due to finite precision we do observe ambiguous situations that may be considered as vertex-vertex contacts, as shown in Fig. 2, and that require special treatment. The difficulty lies in the choice of a common tangent line and two representative points such that the subsequent particle motions under the effect of contact laws at those points does not lead to further mutual intrusion of the particles. The intrusion may increase due to both normal and tangential relative displacements with respect to the four sides involved in the vertex-vertex contact.

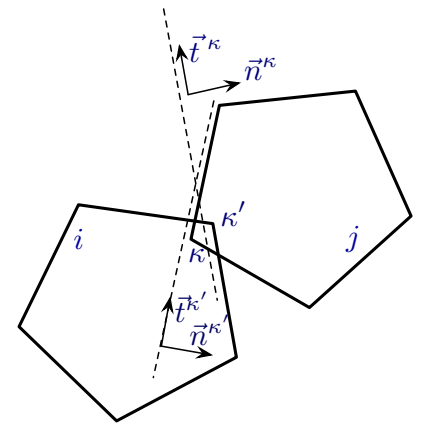

FIG. 2: A vertex-vertex intersection (exagerated) resolved into two side-vertex contacts.

This means that a vertex-vertex contact may be resolved either into two side-vertex contacts or into two side-side contacts and treated as described previously. An example is shown in Fig. 2 where two side-vertex contacts are defined to represent the intersecting vertices (exagerated on the figure). This is a simple and robust method although alternative methods for the choice of the common line and local frame may be proposed.

\section{B. Description of the packings and the simple shear test}

We prepared 13 different packings, each comprising 10000 regular polygons with the same number of sides $n_{s} \in[3,4, . ., 10,11,17,30,40,60]$. Additionally, we build one more packing composed of the same number of disks. The angularity $\alpha=2 \pi / n_{s}$ varies from 0 for disks to $2 \pi / 3$ for triangles. In order to avoid long-range ordering, we introduce size polydispersity by varying the circumradius of the polygons from $0.6\langle d\rangle$ to $2.4\langle d\rangle$, where $\langle d\rangle$ is the mean circumradius, with a uniform distribution by volume fractions.

The particles are initially placed in a semi-periodic box $100\langle d\rangle$ wide, using a geometrical procedure [57, 58]. Next, all packings are sheared by imposing a constant confining stress $\sigma_{\text {wall }}$ and a constant horizontal velocity $v_{\text {wall }}$ on the upper wall, as schematized in Fig. 3. To avoid strain localization at the boundaries and to guarantee that the shear strain is uniformly distributed in the bulk, the particles in contact with the walls are "glued" to them, and the gravity is set to zero. The friction coefficient $\mu_{s}$ between particles is set to 0.4 .

Since we are interested in the quasi-static (rateindependent) behavior, the particle inertia should be negligible compared to the confining pressure. From the shear rate $\dot{\gamma}=v_{\text {wall }} / y_{\text {wall }}$ and $\sigma_{\text {wall }}$ an "inertia parameter" $I$ is defined by [59]

$$
I=\dot{\gamma}\langle d\rangle \sqrt{\frac{\rho}{\sigma_{\text {wall }}}},
$$




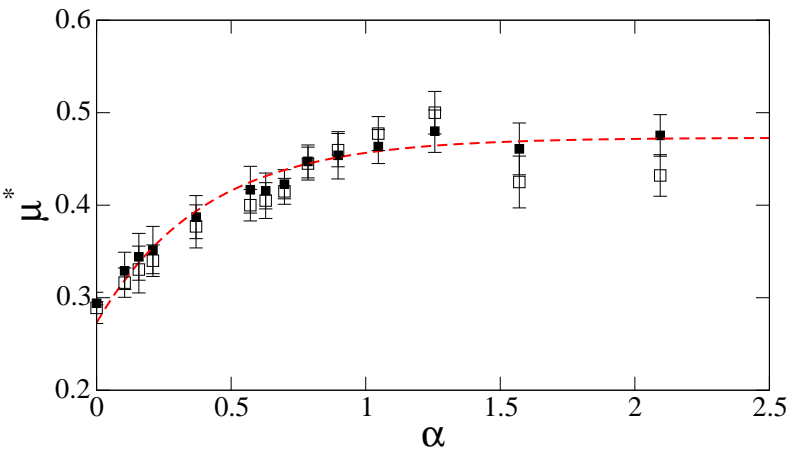

FIG. 5: (Color online) Coefficient of internal friction $\mu^{*}$ as a function of the angularity $\alpha$ evaluated directly from the numerical data (full squares) and predicted by Eq. (8) (empty squares). The error bars represent the standard deviation in the steady state.

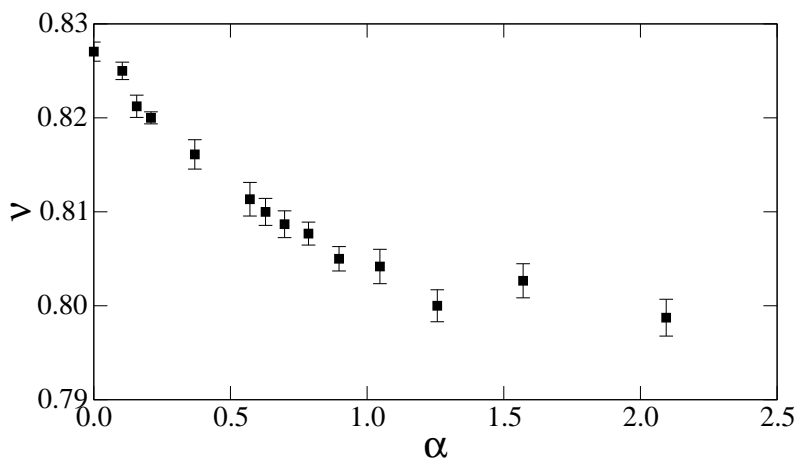

FIG. 6: Steady-state value of the packing fraction $\nu^{*}$ as a function of the angularity $\alpha$. The error bars represent the standard deviation in the steady state.

$\nu_{0}^{*} \simeq 0.828$ (for the disk packing) and saturates to $\nu_{>1}^{*} \simeq$ 0.798. It is remarkable that the packing fraction follows an opposite trend to that of the shear strength. These results represent a new example in which a decrease in packing fraction is accompanied by an increase in shear strength, as it was previously observed for packings of elongated and non-convex particles [18, 22].

In the following, we analyze the microstructural properties of our packings of polygonal particles with the aim of identifying the origins of their shear strength.

\section{MICROMECHANICAL ANALYSIS}

\section{A. Connectivity}

Figure 7 shows a snapshot of the contact network in the steady state for three samples of polygonal particles with $n_{s}=10,5$ and 3 , as well as for the disk packing. We see that the contact network topology varies strongly with angularity. For example, the floating particles are organized in groups in the disk packings whereas they are mostly isolated in the case of triangular particles.
On the other hand, the contact network becomes more connected as the angularity increases. At lowest order, the connectivity of the particles is characterized by the proportion $\kappa$ of non-floating particles and the coordination number $z$ (average number of force-bearing contacts per particle). Figure 8 shows $\kappa$ and $z$ as functions of $\alpha$. We see that $\kappa$ and $z$ decline (from 0.85 to 0.68 and from 3.25 to 3.15 , respectively) as $\alpha$ increases, in accordance with the decrease of packing fraction shown in Fig. 6 . But the trend is reversed beyond $\alpha \simeq 1$ for both $z$ and $\kappa$. In particular, we observe that $z$ increases up to 3.5 which is higher than that in the disk packing. This increase suggests that the sharp corners of very angular particles allow for deep contacts between neighbors that are unreachable for less angular particles. These results show that, for large angularities, the packings are looser but better connected.

The connectivity of the particles may be characterized in more detail by specifying the proportion $P_{c}$ of particles having exactly $c$ contacts. Remark that only the forcebearing contacts are concerned. We have $P_{0}=P_{1}=0$. Figure 9 shows $P_{c}$ for $c=2, \ldots, 8$ as a function of $\alpha$ in the steady state. For all values of $\alpha$, in exception to triangles and squares (i.e. for $\alpha=2 \pi / 3$ and $\alpha=\pi / 2$ in the figure), $P_{3}$ prevails and it remains nearly constant below $\alpha \simeq 1.25$. Beyond $\alpha=1.25$, it declines rapidly contrary to all proportions $P_{c}$ which increase with $\alpha$. We also observe that $P_{4}$ decreases slightly with $\alpha$ for $\alpha<1.25$ whereas in the same range $P_{2}$ increases almost in the same proportion. Hence, as the angularity becomes higher, an increasing number of particles are equilibrated by two opposite forces mostly acting at the sideside contacts. Finally, it is interesting to notice that the proportions $P_{c}$ of particles with more than four contacts remain nearly constant below $\alpha=1.25$ but they increase only slightly in number for squares and triangles. In this way, even a slight increase in angularity (with disk as reference shape) has a strong effect on the connectivity and mechanical behavior as we already remarked with respect to the evolution of $\mu^{*}$ and $\nu^{*}$ in Figs. 5 and 6 .

\section{B. Anisotropy of the contact and force networks}

The shear strength of granular materials is generally attributed to the buildup of an anisotropic structure induced by shearing. This anisotropy is basically related to the distribution of contact normals $\boldsymbol{n}$. Therefore, we may obtain a full description of the state of anisotropy by a partition of various mechanical quantities according to the directions of contact normals. This amounts to replacing the space direction used in continuum mechanics for the representation of the stress and strain fields by the contact orientation.

The most basic descriptor of anisotropy is the probability distribution $P(\boldsymbol{n})$ of the contact normals, which is generically nonuniform. In two dimensions, the unit vector $\boldsymbol{n}$ is described by a single angle $\theta$, and the prob- 


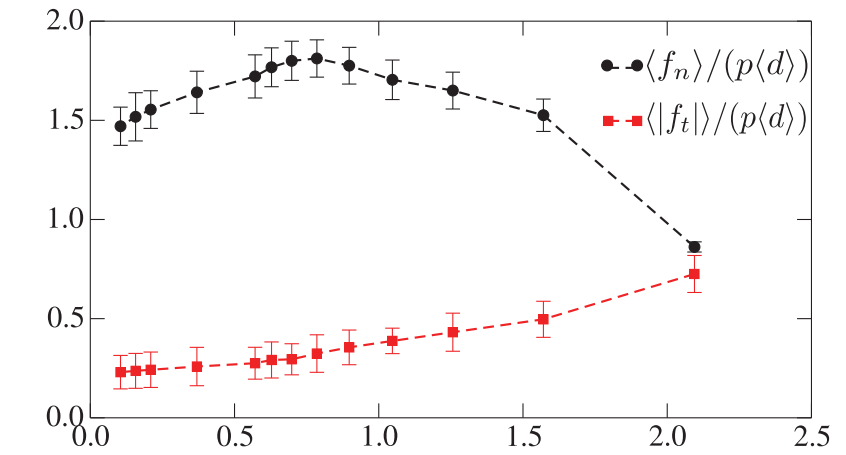

(a)

$\alpha$

(b)

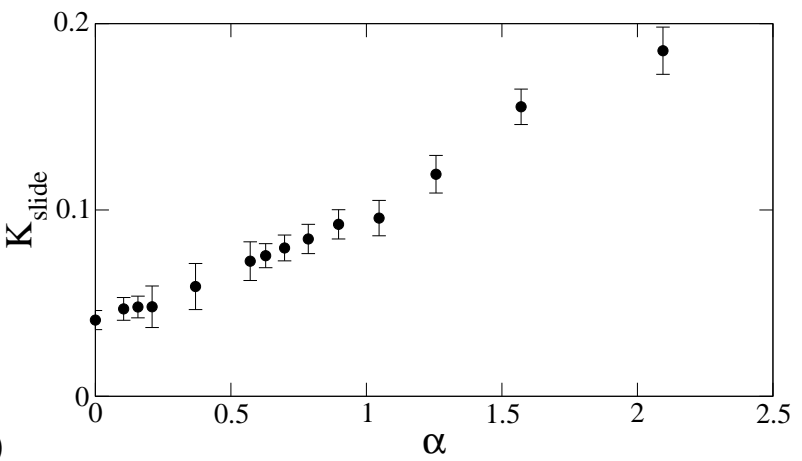

FIG. 12: (Color online) (a) Mean normal force $\left\langle f_{n}\right\rangle$ and mean tangential force $\left\langle\left|f_{t}\right|\right\rangle$ normalized by $p\langle d\rangle$, as functions of the angularity $\alpha$; (b) Proportion $k_{\text {slide }}$ of sliding contacts as a function of $\alpha$. The error bars represent the standard deviation in the steady state.

which $\left.\left|f_{t}\right|=\mu\left|f_{n}\right|\right)$, as functions of $\alpha$. Both $\left\langle f_{n}\right\rangle$ and $\left\langle\left|f_{t}\right|\right\rangle$ initially increase with $\alpha$, but $\left\langle f_{n}\right\rangle$ declines beyond $\alpha \gtrsim 1$ whereas $\left\langle\left|f_{t}\right|\right\rangle$ keeps increasing. The proportion of sliding contacts rises as the particles become increasingly angular and takes values as high as 0.2 , i.e. nearly 4 times above those measured in the packing composed of disks $(\alpha=0)$.

\section{Role of side-to-vertex and side-to-side contacts}

As it was mentioned in the previous subsection, the distinctive features of a material composed of polygonal particles are explained by the possibility of forming side-side contacts. It is thus interesting to investigate the relative roles of the two types of contacts, i.e. sidevertex $(s v)$ and side-side $(s s)$ contacts, with respect to the shear strength and anisotropy. Fig. 13 shows the proportions $k_{s v}$ and $k_{s s}$ of $s v$ and $s s$ contacts, respectively, as a function of $\alpha$. Irrespective of angularity, the $s v$ contacts prevail in the contact network. However, $k_{s v}$ decreases from 1 for the disks $(\alpha=0)$ down to $\simeq 0.75$ for $\alpha \gtrsim 1.25$ and remain practically constant for more angular particles.

Fig. 14 shows a snapshot of the normal force network disk packing as well as three snapshots of the packings with $n_{s}=10,5$, and 3 . The force lines connect particle

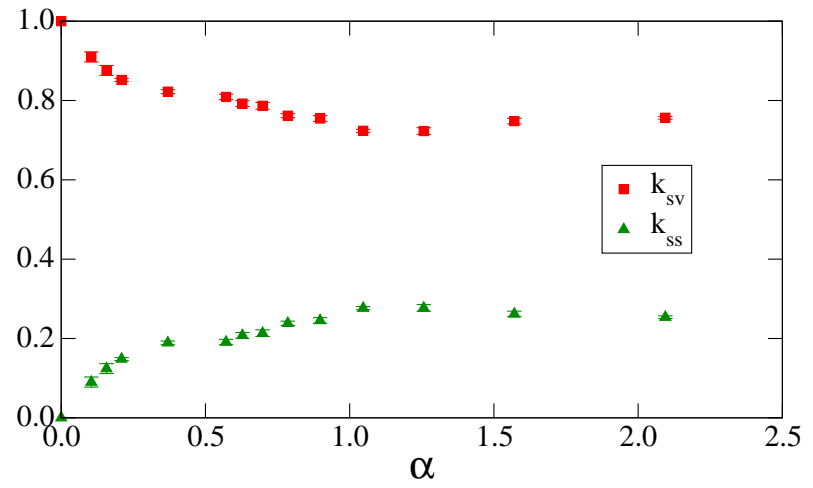

FIG. 13: (Color online) Proportions of side-side $(s s)$ and sidevertex $(s v)$ contacts as functions of the angularity $\alpha$. The error bars represent the standard deviation in the steady state.

centers to the contacts and their thickness is proportional to the normal force. For $n_{s}=10$ and $n_{s}=5$, the $s s$ contacts appear often in distinctive force chains. But for $n_{s}=3$ (triangles), despite approximately the same proportion $k_{s s}$, the $s s$ contact forces are much more diffuse and intricately mixed with $s v$ contacts. This visual impression is consistent with the decrease of $a_{n}$ observed in Fig. 11.

The stress tensor can be partitioned as a sum of two tensors representing the respective contributions of $s v$ and $s s$ contacts by considering the expression (4) of the stress tensor and restricting the summation to each contact type:

$$
\sigma=\sigma_{s v}+\sigma_{s s}
$$

where

$$
\begin{aligned}
& \left(\sigma_{s v}\right)_{i j}=\frac{1}{V} \sum_{c \in \mathcal{A}(s v)} \ell_{i} f_{j}, \\
& \left(\sigma_{s s}\right)_{i j}=\frac{1}{V} \sum_{c \in \mathcal{A}(s s)} \ell_{i} f_{j},
\end{aligned}
$$

where $\mathcal{A}(s v)$ and $\mathcal{A}(s s)$ are the sets of $s v$ and $s s$ contacts, respectively. Fig. 15 displays the evolution of $q / p, q_{s v} / p$, and $q_{s s} / p$ as a function of $\alpha$. It is seen that $q_{s v} / p$ is nearly constant and $\simeq 0.24$, except for the packing of triangular particles in which $q_{s v} / p \simeq 0.35$. In contrast, $q_{s s} / p$ first increases with $\alpha$ from 0 to $\simeq 0.3$ for pentagons and then declines to 0.2 for squares and 0.1 for triangles. This shows that the variation of the shear strength is mostly governed by the contribution of side-side contacts, even if their proportion is low. This profound effect of faceted grain shapes on stress transmission has been previously shown, both experimentally and numerically [17-20].

Along the same lines, we may also evaluate the partial contact and force anisotropies $a_{c \gamma}, a_{l n \gamma}, a_{l t \gamma}, a_{f n \gamma}$, and $a_{f t \gamma}$, where $\gamma$ stands either for $s s$ or for $s v$. Since the privileged directions of the partial angular functions describing the $\gamma$ contacts and forces are practically the same 

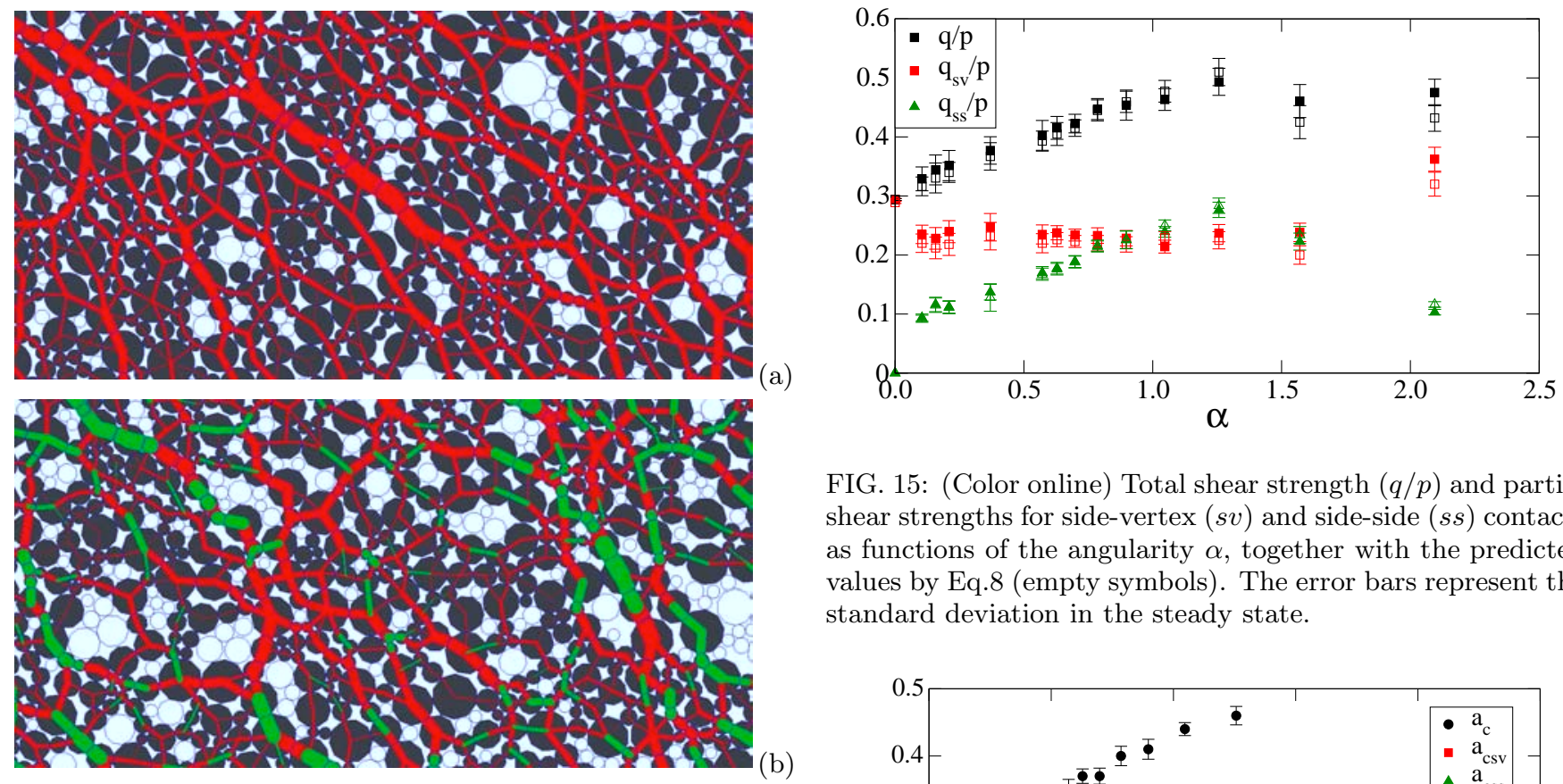

FIG. 15: (Color online) Total shear strength $(q / p)$ and partial shear strengths for side-vertex $(s v)$ and side-side $(s s)$ contacts as functions of the angularity $\alpha$, together with the predicted values by Eq.8 (empty symbols). The error bars represent the standard deviation in the steady state.

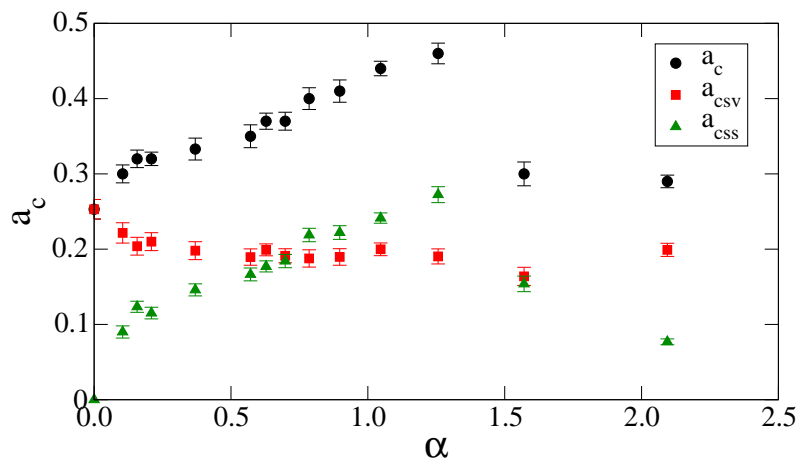

FIG. 16: (Color online) Partial contact orientation anisotropies $a_{c s s}$ and $a_{c s v}$ of $s s$ and $s v$ contacts as functions of the angularity $\alpha$. The error bars represent the standard deviation in the steady state.

and $a_{f t s v} \simeq 0.05$ for all $\alpha$. In other words, the variation of the total anisotropy is mainly governed by that of the anisotropies developed by side-side contacts. The stress plateau discussed previously for the whole contact network for higher angularity is due to the fall-off of $a_{c s s}$ and $a_{\text {fnss }}$ for squares and triangles compensated by the increase of the partial tangential force anisotropy of sideside contacts $a_{f t s s}$. This shows the crucial role of sideside contacts in stress transmission and mobilization of internal friction for most angular particles.

\section{CONCLUDING REMARKS}

as the overall privileged direction for all contacts and forces, the total contact and force anisotropies are given by the sum of the corresponding partial anisotropies. The partial contact and forces anisotropies are shown in Figs. 16 and 17 as a function of $\alpha$ together with the total anisotropies. Note that Eq. (8) is also verified when restricted to $\gamma$ contacts. We see that $a_{c s v} \simeq a_{f n s v} \simeq 0.2$

In this paper, we investigated the effect of particle shape angularity for the quasistatic behavior of sheared granular materials by means of contact dynamics simulations. The particles are regular polygons characterized by their angularity. The macroscopic and microstructural properties of several packings of $10^{4}$ particles in simple shear conditions were analyzed as a function of 


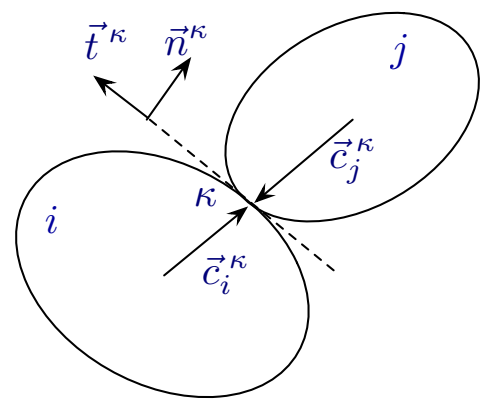

FIG. 18: Geometry of a contact $\kappa$ between two particles $i$ and $j$ with contact vectors $\vec{c}_{i}^{*}$ and $\vec{c}_{j}^{*}$, and contact frame $\left(\vec{n}^{\kappa}, \vec{t}^{\kappa}\right)$.

force may vanish also at an effective contact. In particular, this is the case for $u_{n}=\dot{\delta}_{n}>0$, i.e. for incipient contact opening. Otherwise, the effective contact is persistent and we have $u_{n}=\dot{\delta}_{n}=0$. Hence, Signorini's conditions can be split as follows:

$$
\left\{\begin{array}{l}
\delta_{n}>0 \Rightarrow f_{n}=0 \\
\delta_{n}=0 \wedge\left\{\begin{array}{l}
u_{n}>0 \Rightarrow f_{n}=0 \\
u_{n}=0 \Rightarrow f_{n} \geq 0
\end{array}\right.
\end{array}\right.
$$

We see that for an effective contact, i.e. for $\delta_{n}=0$, Signorini's conditions hold for the variables $u_{n}$ and $f_{n}$.

Like Signorini's conditions, the Coulomb law of dry friction at an effective contact point can be expressed by a set of alternative inequalities for the friction force $f_{t}$ and the sliding velocity $u_{t}$ :

$$
\left\{\begin{array}{l}
u_{t}>0 \Rightarrow f_{t}=-\mu f_{n} \\
u_{t}=0 \Rightarrow-\mu f_{n} \leq f_{t} \leq \mu f_{n} \\
u_{t}<0 \Rightarrow f_{t}=\mu f_{n}
\end{array}\right.
$$

where $\mu$ is the coefficient of friction and it is assumed that the unit tangent vector $\boldsymbol{t}$ points in the direction of the sliding velocity so that $\vec{u}_{t} \cdot \vec{t}=u_{t}$. Like Signorini's conditions, this is a degenerate law that can not be reduced to a (mono-valued) function between $u_{t}$ and $f_{t}$.

Signorini's conditions (Eq. A2) and Coulomb's friction law (Eq. A3) are represented as two graphs in Fig. 19 for an effective contact between two particles. We refer to these graphs as contact laws in the sense that they characterize the relation between relative displacements and forces irrespective of the rheology (visco-elastic or plastic nature) of the particles. These contact laws should be contrasted with force laws (employed in MD simulations), which describe a functional depedence between deformations (attributed to the contact point) and forces that is extracted from the material behavior of the particles. The force laws often employed in MD may also be considered as a "regularization" of the contact laws, in which the vertical branch in Signorini's and Coulomb's graphs is replaced by a steep linear or nonlinear function.
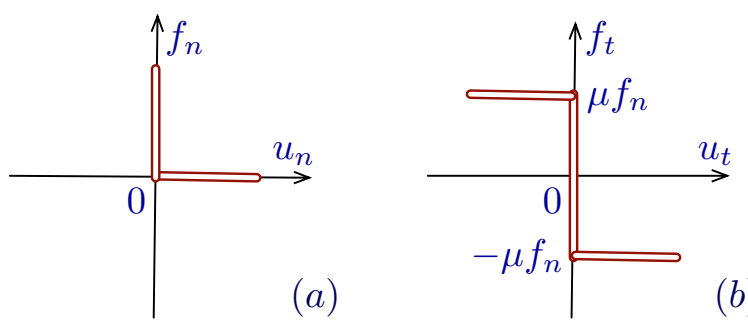

FIG. 19: Graphs of (a) Signorini's conditions and (b) Coulmob's friction law.

\section{Augmented contact laws}

The use of contact laws in the CD method is consistent with the idea of a discrete model defined only at the scale of particle motions and involving no small sub-particle length or force scales inherent to the force laws. But such a "coarse-grained" model of particle motion implies nonsmooth dynamics, i.e. possible discontinuities in particle velocities and forces arising from collisions and variations of the contact status (effective or not, persistent or not, sliding or not). Such events occur frequently in granular flows and hence the approximation of the contact force $f_{n}$ during $\delta t$ is a measure problem in the mathematical sense $[32,54]$. A static or regular force $f^{s}$ is the density of the measure $f^{s} d t$ with respect to time differential $d t$. In contrast, an impulse $p$ generated by a collision has no density with respect to $d t$. In other words, the forces at the origin of the impulse are not resolved at the scale $\delta t$. In practice, however, we can not differentiate these contributions in a "coarse-grained" dynamics, and the two contributions should be summed up to a single measure and the contact force is defined as the average of this measure over $\delta t$.

In a similar vein, the left-limit velocities $u_{n}^{-}$and $u_{t}^{-}$ at time $t$ are not always related by a smooth variation (acceleration multiplied by the time step $\delta t$ ) with the right-limit velocities $u_{n}^{+}$and $u_{t}^{+}$at $t+\delta t$. Hence, we assume that the contact laws (Eq. A2) and (Eq. A3) are satisfied for a weighted mean of the relative left-limit and right-limit velocities:

$$
\begin{aligned}
& u_{n}=\frac{u_{n}^{+}+e_{n} u_{n}^{-}}{1+e_{n}} \\
& u_{t}=\frac{u_{t}^{+}+e_{t} u_{t}^{-}}{1+e_{t}}
\end{aligned}
$$

The physical meaning of the coefficients $e_{n}$ and $e_{t}$ is best illustrated by considering a binary collision between two particles. A binary collision corresponds to an effective contact occuring in the interval $[t, t+\delta t]$ and a persistent contact in the sense of the mean velocity $u_{n}$. In other words, we have $u_{n}=0$ and thus $-u_{n}^{+} / u_{n}^{-}=e_{n}$. Hence, $e_{n}$ can be identified with the normal restitution coefficient. In the same way, for $u_{t}=0$, corresponding to a nonsliding condition (adherence of the two particles 
during their contact), implies $-u_{t}^{+} / u_{t}^{-}=e_{t}$, which is the tangential restitution coefficient. We see that, when Signorini's and Coulomb's graphs are used with the mean velocities given by equation (A12), a contact is persistent in terms of $u_{n}^{+}$(i.e. $u_{n}^{+}=0$ ) only if $e_{n}=0$.

When a collision is not binay, the generated impulses propagate through the contact network so that a contact may experience several successive impulses during $\delta t$. Such events can be resolved for a sufficiently small time increment $\delta t$ or they may be tracked according to an event-driven scheme. The event-tracking strategy is, however, numerically inefficient, of limited applicability and in contradiction with the scope of the CD method based on coarse-grained dynamics. The use of mean velocities (Eq. A12) with the contact laws, should thus be considered as a generalization of restitution coefficients to multiple collisions and contact networks for which the right-limit veocities $u_{n}^{+}$and $u_{t}^{+}$are not simply given by the left-limit velocities multiplied by the coefficients of restitution as in binary collisions but by combining the contact laws with the equations of dynamics.

\section{Nonsmooth motion}

The rigid-body motion of the particles is governed by Newton's equations under the action of imposed external bulk or boundary forces $\vec{F}_{\text {ext }}$, and the contact reaction forces $\vec{f} \kappa$ exerted by neighboring particles at the contact points $\kappa$. An absolute reference frame with unit vectors $(\hat{x}, \hat{y})$ is assumed, and we set $\hat{z}=\hat{x} \times \hat{y}$. Each particle is characterized by its mass $m$, moment of inertia $I$, mass center coordinates $\vec{r}$, mass center velocity $\vec{U}$, angular coordinates $\theta$, and angular velocity $\omega \hat{z}$. For a smooth motion (twice differentiable), the equations of motion of a particle are

$$
\begin{aligned}
m \dot{\vec{U}} & =\vec{F}+\vec{F}_{\text {ext }} \\
I \dot{\omega} & =\mathcal{M}+\mathcal{M}_{\text {ext }}
\end{aligned}
$$

where $\vec{F}=\sum_{\kappa} \vec{f}^{\kappa}$ and $\mathcal{M}=\hat{z} \cdot \sum_{\kappa} \vec{c}^{\kappa} \times \vec{f}^{\kappa}$ where $\vec{c}^{\kappa}$ is the contact vector joining the center of mass to the contact $\kappa$ and $\mathcal{M}_{\text {ext }}$ represents the moment of external forces.

For a nonsmooth motion with time resolution $\delta t$ involving impulses and velocity discontinuities, an integrated form of the equations of dynamics should be used. Hence, the equations of dynamics should be written as an equality of measures:

$$
\begin{aligned}
m d \vec{U} & =d \vec{F}^{\prime}+\vec{F}_{\text {ext }} d t \\
I d \omega & =d \mathcal{M}^{\prime}+\mathcal{M}_{\text {ext }} d t
\end{aligned}
$$

where $d \vec{F}^{\prime}=\sum_{\kappa} d \overrightarrow{f^{\prime} \kappa}$ and $d \mathcal{M}^{\prime}=\hat{z} \cdot \sum_{\kappa} \vec{c}^{\kappa} \times d \overrightarrow{f^{\prime} \kappa}$. These measure differential equations can be integrated over $\delta t$ with the definitions of $\vec{F}$ and $\mathcal{M}$ as approximations of the integral of $d \vec{F}^{\prime}$ and $d \mathcal{M}^{\prime}$. With these definitions, the integration of equation (A7) over $\delta t$ yields

$$
\begin{aligned}
& m\left(\vec{U}^{+}-\vec{U}^{-}\right)=\delta t \vec{F}+\delta t \vec{F}_{\text {ext }} \\
& I\left(\omega^{+}-\omega^{-}\right)=\delta t \mathcal{M}+\delta t \mathcal{M}_{e x t}
\end{aligned}
$$

where $\left(\vec{U}^{-}, \omega^{-}\right)$and $\left(\vec{U}^{+}, \omega^{+}\right)$are the left-limit and rightlimit velocities of the particle, respectively.

The equations of dynamics can be written in a compact form for a set of $N_{p}$ particles by using matrix representation. The particles are labelled with integers $i \in\left[1, N_{p}\right]$. The forces and force moments $F_{x}^{i}, F_{y}^{i}, \mathcal{M}^{i}$ acting on the particles $i$ are arranged in a single highdimensional column vector represented by a boldface letter $\boldsymbol{F}$ belonging to $\mathbb{R}^{3 N_{p}}$. In the same way, the external bulk forces $F_{\text {ext,x }}, F_{\text {ext,y }}, \mathcal{M}_{\text {ext }}$ applied on the particles and the particle velocity components $U_{x}^{i}, U_{y}^{i}, \omega^{i}$ are represented by column vectors $\boldsymbol{F}_{\text {ext }}$ and $\boldsymbol{U}$, respectively. The particle masses and moments of inertia define a diagonal $3 N_{p} \times 3 N_{p}$ matrix denoted by $\boldsymbol{M}$. With these notations, the equations of dynamics (A8) are cast into a single matrix equation:

$$
\boldsymbol{M}\left(\boldsymbol{U}^{+}-\boldsymbol{U}^{-}\right)=\delta t\left(\boldsymbol{F}+\boldsymbol{F}_{\text {ext }}\right)
$$

\section{Transfer equations}

Since the contact laws are expressed in contact variables $\left(u_{n}, u_{t}, f_{n}\right.$ and $\left.f_{t}\right)$, we need to express the equations (A9) in the same variables. The contacts are labelled with integers $\kappa \in\left[1, N_{c}\right]$, where $N_{c}$ is the total number of contacts. Like particle velocities, the contact velocities $u_{n}^{\kappa}$ and $u_{t}^{\kappa}$ can be collected in a column vector $\boldsymbol{u} \in \mathbb{R}^{2 N_{c}}$. In the same way, the contact forces $f_{n}^{\kappa}$ and $f_{t}^{\kappa}$ are represented by a vector $\boldsymbol{f} \in \mathbb{R}^{2 N_{c}}$. We would like to transform the equations of dynamics from $\boldsymbol{F}$ and $\boldsymbol{U}$ to $\boldsymbol{f}$ and $\boldsymbol{u}$. The formal transformation of matrix equations (A9) is straightforward. Since the contact velocities $\boldsymbol{u}$ are linear in particle velocities $\boldsymbol{U}$, the transformation of the velocities is an affine application:

$$
\boldsymbol{u}=\boldsymbol{G} \boldsymbol{U}
$$

where $\boldsymbol{G}$ is a $2 N_{c} \times 3 N_{p}$ matrix containing basically information about the geometry of the contact network. A similar linear application relates $\boldsymbol{f}$ to $\boldsymbol{F}$ :

$$
\boldsymbol{F}=\boldsymbol{H} \boldsymbol{f}
$$

where $\boldsymbol{H}$ is a $3 N_{p} \times 2 N_{c}$ matrix. We refer to $\boldsymbol{H}$ as contact matrix. It contains the same information as $\boldsymbol{G}$ in a dual or symmetric manner. It can easily be shown that $\boldsymbol{H}=$ $\boldsymbol{G}^{T}$ where $\boldsymbol{G}^{T}$ is the transpose of $\boldsymbol{G}$. This property can be inferred from the equivalence between the power $\boldsymbol{F} \cdot \boldsymbol{U}$ developed by "generalized" forces $\boldsymbol{F}$ and the power $\boldsymbol{f} \cdot \boldsymbol{u}$ developed by the bond forces $\boldsymbol{f}$. In general, the matrix $\boldsymbol{H}$ is singular and, by definition, its null space has a dimension at least equal to $2 N_{c}-3 N_{p}$. 
The matrix $H^{i \kappa}$ can be decomposed into two matrices $H_{n}^{i \kappa}$ and $H_{t}^{i \kappa}$ such that

$$
\begin{aligned}
& u_{n}^{\kappa}=\sum_{i} H_{n}^{T, \kappa i} U^{i} \\
& u_{t}^{\kappa}=\sum_{i} H_{t}^{T, \kappa i} U^{i}
\end{aligned}
$$

and

$$
F^{i}=\sum_{\kappa}\left(H_{n}^{i \kappa} f_{n}^{\kappa}+H_{t}^{i \kappa} f_{t}^{\kappa}\right)
$$

Using these relations, the equations (A9) can be trans-

$$
\begin{aligned}
& u_{n}^{\kappa+}-u_{n}^{\kappa-}=\delta t \sum_{i, j} H_{n}^{T, \kappa i} M^{-1, i j}\left\{\sum_{\lambda}\left(H_{n}^{j \lambda} f_{n}^{\lambda}+H_{t}^{j \lambda} f_{t}^{\lambda}\right)+F_{e x t}^{j}\right\}_{n}^{\kappa}=b_{n}^{\kappa}-\left(1+e_{n}\right) \frac{1}{\delta t} u_{n}^{\kappa-}+\left(\frac{\vec{F}_{e x t}^{2_{\kappa}}}{m_{2_{\kappa}}}-\frac{\vec{F}_{e x t}^{1_{\kappa}}}{m_{1_{\kappa}}}\right) \cdot \\
& u_{t}^{\kappa+}-u_{t}^{\kappa-}=\delta t \sum_{i, j} H_{t}^{T, \kappa i} M^{-1, i j}\left\{\sum_{\lambda}^{\lambda}\left(H_{n}^{j \lambda} f_{n}^{\lambda}+H_{t}^{j \lambda} f_{t}^{\lambda}\right)+F_{e x t t_{t}^{j \kappa}}^{j}=b_{t}^{\kappa}-\left(1+e_{t}\right) \frac{1}{\delta t} u_{t}^{\kappa-}+\left(\frac{\vec{F}_{e x t}^{2_{\kappa}}}{m_{2_{\kappa}}}-\frac{\vec{F}_{e x t}^{1_{\kappa}}}{m_{1_{\kappa}}}\right) \cdot \vec{t}(\mathrm{~A}\right.
\end{aligned}
$$

We now can make appear explicitly linear relations between the contact variables from equations (A14) and definitions (A12). We set

$$
\mathcal{W}_{k_{1} k_{2}}^{\kappa \lambda}=\sum_{i, j} H_{k_{1}}^{T, \kappa i} M^{-1, i j} H_{k_{2}}^{j \lambda},
$$

where $k_{1}$ and $k_{2}$ stand for $n$ or $t$. With this notation, equations (A14) can be rewritten as follows:

$$
\begin{aligned}
\frac{1+e_{n}}{\delta t}\left(u_{n}^{\kappa}-u_{n}^{\kappa-}\right) & =\mathcal{W}_{n n}^{\kappa \kappa} f_{n}^{\kappa}+\mathcal{W}_{n t}^{\kappa \kappa} f_{t}^{\kappa} \\
& +\sum_{\lambda(\neq \kappa)}\left\{\mathcal{W}_{n n}^{\kappa \lambda} f_{n}^{\lambda}+\mathcal{W}_{n t}^{\kappa \lambda} f_{t}^{\lambda}\right\} \\
& +\sum_{i, j} H_{n}^{T, \kappa i} M^{-1, i j} F_{e x t}^{j} \\
\frac{1+e_{t}}{\delta t}\left(u_{t}^{\kappa}-u_{t}^{\kappa-}\right)= & \mathcal{W}_{t n}^{\kappa \kappa} f_{n}^{\kappa}+\mathcal{W}_{t t}^{\kappa \kappa} f_{t}^{\kappa} \\
& +\sum_{\lambda(\neq \kappa)}\left\{\mathcal{W}_{t n}^{\kappa \lambda} f_{n}^{\lambda}+\mathcal{W}_{n t}^{\kappa \lambda} f_{t}^{\lambda}\right\} \\
& +\sum_{i, j} H_{t}^{T, \kappa i} M^{-1, i j} F_{e x t}^{j}
\end{aligned}
$$

The coefficients $\mathcal{W}_{k_{1} k_{2}}^{\kappa \kappa}$ for each contact $\kappa$ can be calculated as a function of the contact network geometry and inertia parameters of the two partners $1_{\kappa}$ and $2_{\kappa}$ of the contact $\kappa$. Let $\vec{c}_{i}^{\kappa}$ be the contact vector joining the center of mass of particle $i$ to the contact $\kappa$. The following expressions are easily established:

$$
\begin{aligned}
\mathcal{W}_{n n}^{\kappa \kappa} & =\frac{1}{m_{1_{\kappa}}}+\frac{1}{m_{2_{\kappa}}}+\frac{\left(c_{1 t}^{\kappa}\right)^{2}}{I_{1_{\kappa}}}+\frac{\left(c_{2 t}^{\kappa}\right)^{2}}{I_{2_{\kappa}}} \\
\mathcal{W}_{t t}^{\kappa \kappa} & =\frac{1}{m_{1_{\kappa}}}+\frac{1}{m_{2_{\kappa}}}+\frac{\left(c_{1 n}^{\kappa}\right)^{2}}{I_{1_{\kappa}}}+\frac{\left(c_{2 n}^{\kappa}\right)^{2}}{I_{2_{\kappa}}} \\
\mathcal{W}_{n t}^{\kappa \kappa} & =\mathcal{W}_{t n}^{\kappa \kappa}=\frac{c_{1 n}^{\kappa} c_{1 t}^{\kappa}}{I_{1_{\kappa}}}+\frac{c_{2 n}^{\kappa} c_{2 t}^{\kappa}}{I_{2_{\kappa}}}
\end{aligned}
$$

where $c_{i n}^{\kappa}=\vec{c}_{i}^{\kappa} \cdot \vec{n}^{\kappa}$ and $c_{i t}^{\kappa}=\vec{c}_{i}^{\kappa} \cdot \vec{t}^{\kappa}$ are the components of the contact vectors in the contact frame. The coefficients $\mathcal{W}_{k_{1} k_{2}}^{\kappa \kappa}$ are inverse reduced inertia.
An alternative representation of equations (A16) and (A17) is the following:

$$
\begin{aligned}
& \mathcal{W}_{n n}^{\kappa \kappa} f_{n}^{\kappa}+\mathcal{W}_{n t}^{\kappa \kappa} f_{t}^{\kappa}=\left(1+e_{n}\right) \frac{1}{\delta t} u_{n}^{\kappa}+a_{n}^{\kappa} \\
& \mathcal{W}_{t t}^{\kappa \kappa} f_{t}^{\kappa}+\mathcal{W}_{t n}^{\kappa \kappa} f_{n}^{\kappa}=\left(1+e_{t}\right) \frac{1}{\delta t} u_{t}^{\kappa}+a_{t}^{\kappa}
\end{aligned}
$$

The two offsets $a_{n}^{\kappa}$ and $a_{t}^{\kappa}$ can easily be expressed from the equations (A16) and (A17). The equations (A19) and (A20) or equations (A16) and (A17) are called transfer equations [55]. It is easy to show that

The effect of the approach velocity (left-limit velocity) $\left(u_{n}^{\kappa-}, u_{t}^{\kappa-}\right)$ appears in these equations as an impulse depending on the reduced mass and the restitution coefficient. The effect of contact forces $\vec{f}_{i}^{\lambda}$ acting on the two touching particles $i$ are represented by $b_{n}^{\kappa}$ and $b_{t}^{\kappa}$ given by

$$
\begin{aligned}
b_{n}^{\kappa} & =\frac{1}{m_{2_{\kappa}}} \sum_{\lambda(\neq \kappa)} \vec{f}_{2_{\kappa}}^{\lambda} \cdot \vec{n}^{\kappa}-\frac{1}{m_{1_{\kappa}}} \sum_{\lambda(\neq \kappa)} \vec{f}_{1_{\kappa}}^{\lambda} \cdot \vec{n}^{\kappa}(\mathrm{A} 23) \\
b_{t}^{\kappa} & =\frac{1}{m_{2_{\kappa}}} \sum_{\lambda(\neq \kappa)} \vec{f}_{2_{\kappa}}^{\lambda} \cdot \vec{t}^{\kappa}-\frac{1}{m_{1_{\kappa}}} \sum_{\lambda(\neq \kappa)} \vec{f}_{1_{\kappa}}^{\lambda} \cdot \vec{t}^{\kappa} \cdot(\mathrm{A} 24)
\end{aligned}
$$

The transfer equations (A19) and (A20) define a system of two linear equations between the contact variables at each contact point. The solution, when the values of $a_{n}$ and $a_{t}$ at a contact are assumed, should also verify the contact laws (A2) and (A3). Graphically, this means that the solution is at the intersection between the straight line (A19) and Signorini's graph on one hand, and between (A20) and Coulomb's graph, on the other hand.

\section{Iterative resolution}

In order to solve the system of $2 N_{c}$ transfer equations (in 2D) with the corresponding contact law relations, we proceed by an iterative method which converges to the solution simultaneously for all contact forces and velocities. We first consider a single-contact problem which consists of the determination of contact variables $f_{n}^{\kappa}, f_{t}^{\kappa}$, $u_{n}^{\kappa}$ and $u_{t}^{\kappa}$ at a single contact given the values of the offsets $a_{n}^{\kappa}$ and $a_{t}^{\kappa}$ at the same contact. The solution is given by intersecting the lines representing transfer equations with Signorini's and Coulomb's graphs. The intersection occurs at a unique point due to the positivity of the coefficients $\mathcal{W}_{k_{1} k_{2}}^{\kappa \kappa}$ (positive slope). In other words, the dynamics removes the degeneracy of the contact laws.

Notice, however, that the two intersections can not be established separately when $\mathcal{W}_{n t}^{\kappa \kappa} \neq 0$. To find the local solution, one may consider the intersection of transfer 
equations with the force axis, i.e. by setting $u_{n}=u_{t}=0$. This yields two values $g_{n}^{\kappa}$ and $g_{t}^{\kappa}$ of $f_{n}^{\kappa}$ and $f_{t}^{\kappa}$, respectively:

$$
\begin{aligned}
g_{n}^{\kappa} & =\frac{\mathcal{W}_{t t}^{\kappa \kappa} a_{n}^{\kappa}-\mathcal{W}_{n t}^{\kappa \kappa} a_{t}^{\kappa}}{\mathcal{W}_{n n}^{\kappa \kappa} \mathcal{W}_{t t}^{\kappa \kappa}-\left(\mathcal{W}_{n t}^{\kappa \kappa}\right)^{2}}, \\
g_{t}^{\kappa} & =\frac{\mathcal{W}_{n n}^{\kappa \kappa} a_{n}^{\kappa}-\mathcal{W}_{t n}^{\kappa \kappa} a_{t}^{\kappa}}{\mathcal{W}_{t t}^{\kappa \kappa} \mathcal{W}_{n n}^{\kappa \kappa}-\left(\mathcal{W}_{t n}^{\kappa \kappa}\right)^{2}} .
\end{aligned}
$$

It can be shown that the denominator is positive. If $g_{n}^{\kappa}<$ 0 , then the solution is $f_{n}^{\kappa}=f_{t}^{\kappa}=0$. This corresponds to a breaking contact. Otherwise, i.e. if $g_{n}^{\kappa} \geq 0$, we have $f_{n}^{\kappa}=g_{n}^{\kappa}$. With this value of $f_{n}^{\kappa}$, we can determine the solution of the Coulomb problem. If $g_{t}^{\kappa}>\mu f_{n}^{\kappa}$, the solution is $f_{t}^{\kappa}=\mu f_{n}^{\kappa}$ and in the opposite case, i.e. if $g_{t}^{\kappa}<-\mu f_{n}^{\kappa}$, the solution is $f_{t}^{\kappa}=-\mu f_{n}^{\kappa}$ (sliding contact). Otherwise, i.e. when $-\mu f_{n}^{\kappa}<g_{t}^{\kappa}<\mu f_{n}^{\kappa}$, the solution is $f_{t}^{\kappa}=g_{t}^{\kappa}$ (rolling contact).

In a multicontact system, the terms $b_{n}^{\kappa}$ and $b_{t}^{\kappa}$ in the offsets $a_{n}^{\kappa}$ and $a_{t}^{\kappa}$ depend on the forces and velocities at contacts $\lambda \neq \kappa$; see equations (A21), (A21), (A23) and (A24). Hence, the solution for each contact depends on all other contacts of the system and it must be determined simultaneously for all contacts. An intuitive and robust method to solve the system is to search the solution as the limit of a sequence $\left\{f_{n}^{\kappa}(k), f_{t}^{\kappa}(k), u_{n}^{\kappa}(k), u_{t}^{\kappa}(k)\right\}$ with $\kappa \in\left[1, N_{c}\right]$. Let us assume that the transient set of contact forces $\left\{f_{n}^{\kappa}(k), f_{t}^{\kappa}(k)\right\}$ at the iteration step $k$ is given. From this set, the offsets $\left\{a_{n}^{\kappa}(k), a_{t}^{\kappa}(k)\right\}$ for all contacts can be calculated through the relations (A21) and (A22). The local problem can then be solved for each contact $\kappa$ with these values of the offsets, yielding an updated set of contact forces $\left\{f_{n}^{\kappa}(k+1), f_{t}^{\kappa}(k+1)\right\}$. This correction step is equivalent to the solution of the following local problem:

$$
\begin{aligned}
& \mathcal{W}_{n n}^{\kappa \kappa} f_{n}^{\kappa}(k+1)-\left\{a_{n}^{\kappa}(k)-\mathcal{W}_{n t}^{\kappa \kappa} f_{t}^{\kappa}(k+1)\right\} \stackrel{S}{\longleftrightarrow} f_{n}^{\kappa}(k+1), \\
& \mathcal{W}_{t t}^{\kappa \kappa} f_{t}^{\kappa}(k+1)-\left\{a_{t}^{\kappa}(k)-\mathcal{W}_{n t}^{\kappa \kappa} f_{n}^{\kappa}(k+1)\right\} \stackrel{\leftrightarrow}{\longleftrightarrow} f_{t}^{\kappa}(k+1) .
\end{aligned}
$$

Remark that this force update procedure does not require the contact velocities $\left.u_{n}^{\kappa}(k+1), u_{t}^{\kappa}(k+1)\right\}$ to be calculated as the offsets depend only on the contact forces. The set $\left\{f_{n}^{\kappa}(k), f_{t}^{\kappa}(k)\right\}$ evolves with $k$ by successive corrections and it converges to a solution satisfying the transfer equations and contact laws at all potential contacts of the system. The iteration can be stopped when the set $\left\{f_{n}^{\kappa}(k), f_{t}^{\kappa}(k)\right\}$ is stable with regard to the force update procedure within a prescribed precision criterion $\varepsilon_{f}$ :

$$
\frac{\left|f^{\kappa}(k+1)-f^{\kappa}(k)\right|}{f^{\kappa}(k+1)}<\varepsilon_{f} \quad \forall \kappa .
$$

Finally, from the converged contact forces, the particle velocities $\left\{\vec{U}^{i}\right\}$ can be computed by means of the equations of dynamics (A8).

The iterative procedure depicted above provides a robust method which proves efficient in the context of granular dynamics. The information is treated locally and no large matrices are manipulated during iterations. The number $N_{i}$ of necessary iterations to converge is strongly dependent on the precision $\varepsilon_{f}$ but not on $\delta t$. The number of iterations is substantially reduced when the iteration is initialized with a globally correct guess of the forces. This is the case when the forces at each time step are initialized with the forces computed in the preceding step.

The uniqueness of the solution in a multicontact system with rigid particles is not guaranteed at each step of evolution. We have $3 N_{p}$ equations of dynamics and $2 N_{c}$ contact relations. The unknowns of the problem are $3 N_{p}$ particle velocities and $2 N_{c}$ contact forces. The indeterminacy arises from the fact that the $2 N_{c}$ contact relations are inequations. Thus, the extent of indeterminacy of the solution reflects all possible combinations of contact forces accommodating those contact relations. The degree of indeterminacy may be high, but it does not imply significant force variability since the solutions are strongly restrained by the contact laws. In practice, the issue is more to find a mechanically admissible solution (verifying the contact laws and equations of dynamics) than indeterminacy. In other words, the variability of the solution is often below the precision controlled by $\varepsilon_{f}$ when the forces are computed at each time step from the forces at the preceding step.

\section{Time-stepping scheme}

In CD method, the global problem of the determination of forces and velocities, as described above, is associated with a time-stepping scheme. This scheme is based on the fact that the first condition of Signorini's relations in (A2) is the only condition referring to space coordinates. Both the equations of dynamics and contact laws are formulated at the velocity level, and the first condition of Signorini is accounted for by considering only the effective contacts where $\delta_{n}=0$. Hence, the contact network is defined explicitly from particle positions and it will no more evolve during the time interval $\delta t$. But the treatment of forces and velocities is fully implicit, and the right-limit velocities $\left\{\vec{U}^{i+}, \omega^{i+}\right\}$ should be used to increment particle positions.

These remarks devise the following time-stepping scheme. Let $t$ and $t+\delta t$ be the considered time interval. The configuration $\left\{\vec{r}^{i}(t)\right\}$ and particle velocities $\left\{\vec{U}^{i}(t), \omega^{i}(t)\right\}$ are given at time $t$. The latter play the role of left-limit velocities $\left\{\vec{U}^{i-}, \omega^{i-}\right\}$. The contact network $\left\{\kappa, \vec{n}^{\kappa}, \vec{t}^{\kappa}\right\}$ is set up from the configuration at time $t$ or from an intermediate configuration $\left\{\vec{r}_{m}^{i}\right\}$ defined by

$$
\vec{r}_{m}^{i} \equiv \vec{r}^{i}(t)+\frac{\delta t}{2} \vec{U}^{i}(t) .
$$

When this configuration is used for contact detection, other space-dependent quantities such as the inverse mass parameters $\mathcal{W}_{k_{1} k_{2}}^{\kappa \kappa}$ and external forces $\vec{U}_{\text {ext }}^{i}$ should consistently be defined for the same configuration and at the same time $t+\delta t / 2$. Then, the forces and velocities are iteratively determined for the contact network and the right-limit particle velocities $\left\{\vec{U}^{i+}, \omega^{i+}\right\}$ are calculated. The latter correspond to the velocities at the end of the time step $t+\delta t$ :

$$
\begin{aligned}
\vec{U}^{i}(t+\delta t) & =\vec{U}^{i+}, \\
\omega^{i}(t+\delta t) & =\omega^{i+} .
\end{aligned}
$$

Finally, the positions are updated by integrating the updated velocities:

$$
\begin{aligned}
& \vec{r}^{i}(t+\delta t)=\vec{r}_{m}^{i}+\frac{\delta t}{2} \vec{U}^{i}(t+\delta t), \\
& \theta^{i}(t+\delta t)=\theta_{m}^{i}+\frac{\delta t}{2} \omega^{i}(t+\delta t) .
\end{aligned}
$$


This scheme is unconditionally stable due to its inherent implicit time integration. Hence, no damping parameters at any level are needed. For this reason, the time step $\delta t$ can be large. The real limit imposed on the time step is cumulative round-off errors in particle positions since the latter are updated from the integration of the velocities. Although the excessive overlaps have no dynamic effect in the CD method, they falsify the geometry and thus the evolution of the system. A sufficiently high precision or a large enough number of iterations is required to avoid such errors. The time step is not a precision parameter but a coarse-graining parameter for nonsmooth dynamics. It should be reduced if the impulse dynamics at small time scales is of interest.
[1] K. Mair, K. Frye, and C. Marone, Journal Of Geophysical Research 107 (2002).

[2] F. Alonso-Marroquin and H. J. Herrmann, Phys. Rev. E 66, 021301 (2002).

[3] A. Mirghasemi, L. Rothenburg, and E. Maryas, Geotechnique 52, N 3, 209 (2002).

[4] C. Nouguier-Lehon, B. Cambou, and E. Vincens, Int. J. Numer. Anal. Meth. Geomech 27, 1207 (2003).

[5] Y. Guo and J. Morgan, Journal Of Geophysical Research 109 (2004).

[6] J. L. Anthony and C. Marone, Journal Of Geophysical Research 110 (2005).

[7] A. Pena, A. Lizcano, F. Alonso-Marroquin, and H. Herrman, Int. J. For Numer. Anal. Meth. Geomech. 00, 1 (2006).

[8] E. Azéma, F. Radjai, R. Peyroux, and G. Saussine, Phys. Rev. E 76, 011301 (2007).

[9] S. A. Galindo-Torres, J. D. Munoz, and F. AlonsoMarroquin, Phys Rev E 82, 056713 (2010).

[10] E. Azéma, F. Radjai, and G. Saussine, Mechanics of Materials 41, 721 (2009).

[11] E. Azéma, F. Radjai, R. Peyroux, F. Dubois, and G. Saussine, Phys. Rev. E 74, 031302 (2006).

[12] E. Azéma, Y. Descantes, N. Roquet, J.-N. Roux, and F. Chevoir, Phys. Rev. E accepted, ?? (2012).

[13] A. Donev, F. Stillinger, P. Chaikin, and S. Torquato, Phys Rev Lett. 92, 255506 (2004).

[14] S. Sacanna, L. Rossi, A. Wouterse, and A. P. Philipse, J. Phys.: Condens. Matter 376108, 16p (2007).

[15] I. Zuriguel, T. Mullin, and J. Rotter, Phys. Rev. Lett. 98, 028001 (2007).

[16] I. Zuriguel and T. Mullin, Proc. R. Soc. A 464, 99 (2008).

[17] R. C. Hidalgo, I. Zuriguel, D. Maza, and I. Pagonabarraga, Phys. Rev. Lett. 103, 118001 (2009).

[18] E. Azéma and F. Radjaï, Phys Rev E 81, 051304 (2010).

[19] T. Kanzaki, M. Acevedo, I. Zuriguel, I. Pagonabarraga, D. Maza, and R. Hidalgo, Eur. Phys. J. E 34, 133 (2011).

[20] E. Azéma and F. Radjai, Phys Rev E 85, 031303 (2012).

[21] B. Saint-Cyr, C. Voivret, J. Delenne, F. Radjai, and P. Sornay, in Conference Information: 6th International Conference on the Micromechanics of Granular Media,POWDERS AND GRAINS (2009), vol. 1145 of AIP Conference Proceedings, pp. 389-392.

[22] B. Saint-Cyr, C. Voivret, J.-Y. Delenne, F. Radjai, and P. Sornay, Phys Rev E 84, 041302 (2011).

[23] G. Saussine, C. Cholet, P. Gautier, F. Dubois, C. Bohatier, and J. Moreau, Comput. Methods Appl. Mech. Eng. 195, 2841 (2006).

[24] E. Nezami, Y. Hashash, D. Zaho, and J. Ghaboussi, Int. J. Numer. Anal. Meth. Geomech. 30, 783 (2006).

[25] F. Radjai and F. Dubois, eds., Discrete Numerical Modeling of Granular Materials (Wiley-ISTE,
New-York, March 2011), iSBN: 978-1-84821-260-2, URL http://eu.wiley.com/WileyCDA/WileyTitle/ productCd-1848212607.html.

[26] E. Azéma, F. Radjai, R. Peyroux, V. Richefeu, and G. Saussine, Eur. Phys. J. E 26, 327 (2008).

[27] J. J. Moreau, Journal of differential equations 26, 347 (1977).

[28] J. J. Moreau, Comptes Rendus de l'Académie des Sciences 296, 1473 (1983).

[29] J. J. Moreau, in Topics in Nonsmooth Mechanics, edited by P. Panagiotopoulos and G. Strang (Bikhäuser, Basel, 1988), pp. 1-74.

[30] J. J. Moreau, in CISM (1988), pp. 1-82.

[31] J. J. Moreau, in Powders 8 Grains 93 (A. A. Balkema, Rotterdam, 1993), p. 227.

[32] J. Moreau, European J. Mech. A Solids 13, 93 (1994).

[33] M. Jean and E. Pratt, International Journal Eng. Sci. pp. 497-513 (1985).

[34] M. Jean, Arch. of Mech., Warszawa 40, 677 (1988).

[35] M. Jean and J. J. Moreau, in Proceedings of Contact Mechanics International Symposium (Presses Polytechniques et Universitaires Romandes, Lausanne, Switzerland, 1992), pp. 31-48.

[36] M. Jean, F. Jourdan, and B. Tathi, in Proc. of IDDRG 1994 (1994), Handbooks on Theory and Engineering Applications of Computational Methods.

[37] M. Jean, Frictional contact in rigid or deformable bodies: numerical simulation of geomaterials (Elsevier Science Publisher, Amsterdam, 1995), pp. 463-486.

[38] M. Jean, Computer Methods in Applied Mechanic and Engineering 177, 235 (1999).

[39] P. Cundall, in Proceedings of the Symposium of the International Society of Rock Mechanics (1971), Vol. 1, 132150.

[40] P. A. Cundall and O. Strack, geotechnique 29, 47 (1979).

[41] C. Thornton and K. K. Yin, Powder Technol. 65, 153 (1991).

[42] H. J. Herrmann, in The first Nisshin Engineering Particle Technology International Seminar: Discrete Particle Simulations in Powder Technology (Osaka, Japan, 1993), p. 8.

[43] C. Thornton, in The first Nisshin Engineering Particle Technology International Seminar: Discrete Particle Simulations in Powder Technology (Osaka, Japan, 1993), p. 17.

[44] T. Pöschel and V. Buchholtz, J. Phys. I France 5, 1431 (1995).

[45] C. Thornton, Journal of Applied Mechanics 64, 383 (1997).

[46] S. Luding, in Physics of dry granular media - NATO ASI Series E350, edited by H. J. Herrmann, J.-P. Hovi, and S. Luding (Kluwer Academic Publishers, Dordrecht, 
1998), p. 285.

[47] S. McNamara and H. Herrmann, Phys. Rev. E 70, 061303 (2004).

[48] X. Garcia and E. Medina, Phys Rev E Stat Nonlin Soft Matter Phys 75, 061308 (2007).

[49] F. A. Gilabert, J.-N. Roux, and A. Castellanos, Phys. Rev. E 75, 011303 (2007).

[50] V. Richefeu, M. S. El Youssoufi, R. Peyroux, and F. Radjai, International Journal for Numerical and Analytical Methods in Geomechanics 9999, n/a (2007), URL http://dx.doi.org/10.1002/nag.674.

[51] V. Visseq, A. Martin, D. Iceta, E. Azéma, D. Dureisseix, and P. Alart, Comput Mech DOI 10.1007/s00466012-0699-5 (2012).

[52] H.-G. Matuttis, S. Luding, and H. J. Herrmann, Powder Technology 109, 278 (2000).

[53] F. Radjaï and V. Richefeu, Mechanics of Materials 41, 715 (2009).

[54] J. Moreau, in Novel approaches in civil engineering, edited by M. Frémond and F. Maceri (Springer-Verlag, Berlin, 2004), vol. 14 of Lecture Notes in Applied and Computational Mechanics, pp. 1-46.

[55] F. Radjaï and E. Azéma, Eur. J. Env. Civil Engineering 13, 204 (2009).

[56] F. Dubois and M. Jean, in Analysis and Simulation of Contact Problems, edited by P. Wriggers and U. Nackenhorst (Springer Berlin / Heidelberg, 2006), vol. 27 of Lecture Notes in Applied and Computational Mechanics, pp. 375-378, 10.1007/3-540-31761-9 44, URL http: //dx.doi.org/10.1007/3-540-31761-9_44.

[57] A. Taboada, K. J. Chang, F. Radjai, and F. Bouchette, Journal Of Geophysical Research 110, 1 (2005).

[58] C. Voivret, F. Radjai, J.-Y. Delenne, and M. S. E. Youssoufi, Phys Rev E 76, 021301 (2007).
[59] GDR-MiDi, Eur. Phys. J. E 14, 341 (2004).

[60] J. Mitchell and K. Soga, Fundamentals of Soil Behavior (Wiley, New-York, NY, 2005).

[61] J. J. Moreau, in Friction, Arching, Contact Dynamics, edited by D. E. Wolf and P. Grassberger (World Scientific, Singapore, 1997), pp. 233-247.

[62] M. Oda, J. Koshini, and S. Nemat-Nasser, Geotechnique 30, 479 (1980).

[63] L. Rothenburg and R. J. Bathurst, Geotechnique 39, 601 (1989).

[64] F. Radjai, D. E. Wolf, M. Jean, and J. Moreau, Phys. Rev. Letter 80, 61 (1998).

[65] F. Radjai, H. Troadec, and S. Roux, in Granular Materials: Fundamentals and Applications, edited by S. Antony, W. Hoyle, and Y. Ding (RS.C, Cambridge, 2004), pp. 157-184.

[66] B. Cambou, P. Dubujet, and C. Nouguier-Lehon, Mechanics of Materials 36, 1185 (2004).

[67] C. Nouguier-Lebon, E. Vincens, and B. Cambou, International journal of Solids and Structures 42, 6356 (2005).

[68] N. Estrada, A. Taboada, and F. Radjai, Phys Rev E 78, 021301 (2008).

[69] C. Voivret, F. Radjai, J.-Y. Delenne, and M. S. E. Youssoufi, Phys. Rev. Lett. 102, 178001 (2009).

[70] N. Estrada, E. Azéma, F.Radjai, and A. Taboada, Phys Rev E 84, 011306 (2011).

[71] F. Radjai, J.-Y. Delenne, E. Azéma, and S. Roux, Granular Matter pp. DOI 10.1007/s10035-012-0321-8 (2012).

[72] H. Ouadfel and L. Rothenburg, Mechanics of Materials 33, 201 (2001).

[73] A similar relation can be obtained in 3D using spherical harmonics [10, 72]. 